\title{
The psychological effects of nursing interventions on patients with suspected COVID-19 during isolation
}

\author{
Weiwei Niu ${ }^{1 \#}$, Xinhong Ma ${ }^{2 \#}$, Ying Zhang ${ }^{3}$, Zengfang Sun ${ }^{4}$ \\ ${ }^{1}$ Department of Isolation Ward, Tianjin Third Central Hospital, Tianjin Key Laboratory of In-vitro Life Support for Severe Diseases, Tianjin \\ Research Center of Artificial Cell Engineering Technology, Tianjin Institute of Hepatobiliary Diseases, Tianjin, China; ${ }^{2}$ Department of Fever \\ Outpatients, The First Affiliated Hospital of Tianjin University of Chinese Medicine, Tianjin, China; ${ }^{3}$ Nursing Department of Tianjin Third Central \\ Hospital, Tianjin, China; ${ }^{4}$ Department of Thoracic Surgery, Tianjin Third Central Hospital, Tianjin, China \\ Contributions: (I) Conception and design: W Niu, X Ma; (II) Administrative support: W Niu; (III) Provision of study materials or patients: W Niu, X \\ Ma; (IV) Collection and assembly of data: All authors; (V) Data analysis and interpretation: X Ma; (VI) Manuscript writing: All authors; (VII) Final \\ approval of manuscript: All authors. \\ \#These authors contributed equally to this work. \\ Correspondence to: Prof. Weiwei Niu. Department of Isolation Ward, Tianjin Third Central Hospital, Tianjin Key Laboratory of In-vitro Life Support \\ for Severe Diseases, Tianjin Research Center of Artificial Cell Engineering Technology, Tianjin Institute of Hepatobiliary Diseases, Tianjin 300170, \\ China. Email: tjwei81@163.com.
}

Background: To investigate the psychological characteristics of patients with suspected coronavirus disease 2019 (COVID-19) during isolation and to evaluate the effects of psychological nursing interventions.

Methods: A total of 137 patients with suspected COVID-19 were assessed using the 7-item Generalized Anxiety Disorder Scale (GAD-7), the 9-item Patient Health Questionnaire (PHQ-9), and the Trait Coping Style Questionnaire (TCSQ). Psychological nursing intervention measures were implemented, after which the patients were re-evaluated.

Results: Before intervention, the rates of anxiety and depression among the patients with suspected COVID-19 were $46.72 \%$ and $48.20 \%$, respectively. After intervention, statistically significant reductions were observed in the GAD-7 and PHQ-9 scores (both $\mathrm{P}<0.05$ ). Before intervention, the TCSQ positive and negative coping scores were $31.07 \pm 5.25$ and $28.78 \pm 6.72$, respectively, compared with $36.40 \pm 5.93$ and $24.60 \pm 5.99$, respectively, after intervention; these differences were statistically significant (both $\mathrm{P}<0.01$ ). Before and after intervention, the patients' GAD-7 and PHQ-9 scores were negatively correlated with the positive coping score but positively correlated with the negative coping score.

Conclusions: For COVID-19 or similar public health emergencies, timely and flexible appropriate psychological intervention and counseling is crucial. Correct implementation of psychological intervention methods can effectively relieve patients' negative emotions, and play an important role in assisting patients during the isolation period.

Keywords: Coronavirus disease 2019 (COVID-19); suspected patients; isolation; psychological care

Submitted Jan 14, 2021. Accepted for publication Apr 17, 2021.

doi: $10.21037 /$ apm-21-516

View this article at: http://dx.doi.org/10.21037/apm-21-516

\section{Introduction}

Coronavirus disease 2019 (COVID-19) is currently spreading around the globe (1). It is a class B acute respiratory infectious disease resulting from infection with severe acute respiratory syndrome coronavirus 2 (SARS-COV-2) (2). With rapid transmission and strong infectivity, COVID-19 quickly became a pandemic (3).

Designated fever clinics are one of the main modes of screening for COVID-19. As one of the designated fever 
clinics in Tianjin, China, Tianjin Third Central Hospital has set up an isolation ward to provide patients suspected with COVID-19 with sampling and clinical treatment, as well as a place to await their pharyngeal test results.

In the face of the sudden epidemic, isolated patients not only have physical problems, but they are also prone to anxiety, depression, and other psychological problems (4). Patients in isolation not only hope to receive the best treatment for their diagnosis, but they also hold high expectations and demands for psychological care $(5,6)$. Therefore, to ensure its patients are supported successfully throughout the isolation treatment period, Tianjin Third Central Hospital provides psychological nursing care.

In this paper, the psychological characteristics of patients with suspected COVID-19 infection were analyzed during isolation. Subsequently, nursing interventions were implemented to relieve the psychological pressure felt by the patients in order to provide a basis for follow-up clinical nursing research and ensure the safety of patients during isolation.

This study used the General Anxiety Scale (GAD-7 score), the Patient Health Questionnaire Depression Scale (PHQ-9 score), and the Trait Coping Style Questionnaire (TCSQ score) to conduct psychological evaluations of 137 suspected COVID-19 patients, and take corresponding measures. After the intervention of psychological nursing measures, the patient's mental state was evaluated again, and it was found that the above several scales can be used to evaluate the mental state of isolated patients, and the corresponding nursing measures can improve the patient's mental state. This study provides a new theoretical basis for the evaluation measures of the mental state of isolated patients. We present the following article in accordance with the STROBE reporting checklist (available at http:// dx.doi.org/10.21037/apm-21-516).

\section{Methods}

\section{Patients}

Patients with suspected COVID-19 infection who were admitted to the isolation ward of Tianjin Third Central Hospital between January 25 and February 29, 2020 participated in this research. The inclusion criteria for patients were: (I) suspected with COVID-19 and admitted to the isolation ward during the epidemic; (II) aged $\geq 15$ years old; (III) able to communicate clearly with no mental impairment; (IV) volunteered to participate in the investigation. The exclusion criteria were: (I) patients with a previous diagnosis of mental illness; (II) patients who were physically weak and unable to complete the questionnaire. All procedures performed in this study involving human participants were in accordance with the Declaration of Helsinki (as revised in 2013). This study has been approved by the Ethics Committee of Tianjin Third Central Hospital (No. bd2020111), and all enrolled patients signed informed consent.

\section{Research tools}

\section{Generalized Anxiety Disorder-7 (GAD-7)}

The GAD-7 scale consists of 7 items designed to gauge the extent to which patients suffer from feelings such as nervousness and worry. Each item is scored on a scale of 0 to 3 points $(0=$ none at all, $1=$ a few days, $2=$ more than a week, $3=$ almost every day), with a total score of 0 to 21 points. The severity of psychological distress is classified according to 4 standard thresholds: minimal or none (0 to 4 points), mild (5 to 9 points), moderate (10 to 14 points), and severe ( $\geq 15$ points). This scale has high reliability and validity, and is suitable for a wide variety of people.

\section{Patient Health Questionnaire-9 (PHQ-9)}

The PHQ-9 comprises 9 items and is used to assess the frequency at which patients experience depressive symptoms. Each item is scored on a scale of 0 to 3 points $(0=$ none, $1=$ a few days, $2=$ more than a week, $3=$ almost every day), with a total score range of 0 to 27 points. The severity of psychological distress is classified according to the standard thresholds: minimal or none (0 to 4 points), mild (5 to 9 points), moderate (10 to 14 points), and severe ( $\geq 15$ points). This scale has been proved to have high reliability and validity in various Chinese populations.

\section{Trait Coping Style Questionnaire (TCSQ)}

The TCSQ assesses positive coping and negative coping styles across 20 items. The questionnaire uses a 1 to 5 point scale to reflect the stable coping styles related to personality traits of an individual.

\section{Survey methods}

Patients gained access to the psychological evaluation questionnaire by scanning a QR code within 4 hours of admission (before intervention) and before discharge (after intervention). 


\section{Interventions}

Nursing staff in the isolation ward implemented psychological nursing interventions for patients admitted to the ward with suspected COVID-19 infection. Interventions included: (I) taking care to understand patients, and explaining to patients the importance and necessity of isolation screening, in order to improve their understanding and degree of cooperation; (II) patiently introducing the layout of the isolation ward, isolation rules, scope of activities, and matters requiring attention to patients; (III) providing patients with targeted treatment and life care; (IV) making patients aware of the importance of using protective equipment, reducing patients' fear, and establishing a good nurse-patient relationship through health education; (V) providing COVID-19-related health education materials to guide patients in gaining an accurate understanding of the disease and improve patients' ability to take care of themselves and their health; (VI) using a telephone, WeChat, or other forms of communication to establish contact with patients in order to help patients understand and address their psychological problems, and to promote a positive attitude and positive and effective actions. (VII) helping patients to clear up anxiety, depression, and other negative emotions; (VIII) using non-verbal communication skills to communicate between nurses and patients while keeping spatial distance, such as through the eyes, movements, and text communication (e.g., "thumbs up" to show encouragement, the "win" sign to show when a patient was getting better, or a pat on the shoulder with the back of the hand to express comfort when a patient is feeling sad; (IX) taking the initiative to help elderly or weak individuals, children, or other patients are unable to take care of themselves; $(\mathrm{X})$ Helping isolated patients to establish social emotional support, implemented by responsible nurses with rich experience, strong communication and coordination skills, actively persuading family members to increase communication and contact with patients, giving patients psychological comfort, eliminating their worries, and guiding patients to take the initiative communicate with the outside world and improving the social support system for each patient; (XI) guiding patients to use emotional regulation auxiliary intervention methods, including attentional shifting, physical decompression, breathing relaxation, self-education, and communication regulation.

\section{Statistical methods}

SPSS22.0 statistical software (IBM Corporation, Armonk, NY, USA) was used to collate and analyze the data. Measurement data were expressed as mean \pm standard deviation $(\bar{x} \pm \mathrm{S})$. Comparisons between 2 groups were performed using $t$-tests. The correlation between 2 variables was analyzed by Pearson's linear correlation. Twosided tests were used, and $\mathrm{P}<0.05$ represented a statistically significant difference.

\section{Results}

\section{The general data of patients with suspected COVID-19}

A total of 137 patients, including 64 males and 73 females, were eventually included in the study. The patients ranged in age from $15-88$ years old $(43.3 \pm 15.8$ years old) and were isolated for $1-11$ days $(2.7 \pm 1.1$ days $)$. Finally, 7 patients were diagnosed with COVID-19.

The details of age, gender, marital status, occupation, education level and days of hospitalization are shown in Table 1.

\section{Comparison of GAD-7 score and $P H Q-9$ score before and after psychological nursing intervention}

Of the 137 patients with suspected COVID-19 who were enrolled in this study, $73(53.28 \%)$ patients had no obvious anxiety, 42 (30.66\%) patients had mild anxiety, and $22(16.06 \%)$ patients had moderate to severe anxiety, according to their GAD-7 scores. Before intervention, the GAD-7 scores of the patients with no obvious anxiety, mild anxiety, and mild to severe anxiety were $1.75 \pm 1.48$, $7.26 \pm 1.18$, and $13.86 \pm 2.70$, respectively. After intervention, the GAD-7 scores of the 3 groups were $1.45 \pm 0.85$, $4.31 \pm 1.15$, and $8.36 \pm 3.26$, respectively, and the difference from before intervention was statistically significant. Before intervention, the PHQ-9 showed that 71 (51.82\%) patients had no obvious depression, 49 (35.77\%) patients had mild depression, and 17 (12.41\%) patients had moderate to severe depression. The PHQ-9 scores in the non-depressed, mild, and moderate to severe depression groups were $2.38 \pm 1.31,6.86 \pm 1.24$, and $13.82 \pm 3.52$, respectively, compared with $1.38 \pm 0.82,3.33 \pm 1.71$, and $7.76 \pm 3.42$, respectively, after intervention. The difference between the 
Table 1 General data of patients with suspected COVID-19 (N=137)

\begin{tabular}{|c|c|c|}
\hline Characteristic & $\mathrm{N}$ & $\%$ \\
\hline \multicolumn{3}{|l|}{ Sex } \\
\hline Male & 64 & 46.7 \\
\hline Female & 73 & 53.3 \\
\hline \multicolumn{3}{|l|}{ Age (years) } \\
\hline$<20$ & 7 & 5.1 \\
\hline 20-29 & 16 & 11.7 \\
\hline 30-39 & 44 & 32.1 \\
\hline $40-49$ & 24 & 17.5 \\
\hline$>50$ & 46 & 33.6 \\
\hline \multicolumn{3}{|l|}{ Marital status } \\
\hline Unmarried & 22 & 16.1 \\
\hline Married & 97 & 70.8 \\
\hline Divorce & 12 & 8.8 \\
\hline Widowed & 6 & 4.4 \\
\hline \multicolumn{3}{|l|}{ Occupational status } \\
\hline Student & 16 & 11.7 \\
\hline Employed & 81 & 59.1 \\
\hline Retired & 21 & 15.3 \\
\hline Unemployed & 19 & 13.9 \\
\hline \multicolumn{3}{|l|}{ Education level } \\
\hline Junior high school or below & 30 & 21.9 \\
\hline College or below & 42 & 30.7 \\
\hline Bachelor's degree & 57 & 41.6 \\
\hline Master's degree or above & 8 & 5.8 \\
\hline \multicolumn{3}{|c|}{$\begin{array}{l}\text { Days of hospitalization (including } \\
\text { before transfer) }\end{array}$} \\
\hline $1-3$ days & 117 & 85.4 \\
\hline $4-6$ days & 18 & 13.1 \\
\hline $7-10$ days & 2 & 1.5 \\
\hline
\end{tabular}

COVID-19, coronavirus disease 2019.

2 groups was statistically significant (Table 2).

\section{Comparison of TCSQ score before and after psychological nursing intervention}

Before intervention, the patients had a TCSQ positive coping score (PC score) of $31.07 \pm 5.25$ and a negative coping score (NC score) of $28.78 \pm 6.72$. After intervention, the PC and NC scores were $36.40 \pm 5.93$ and $24.60 \pm 5.99$, respectively. The differences in the preand post-intervention scores were statistically significant (Table 3).

\section{Correlation analysis of GAD-7 total score, PHQ-9 total score and TCSQ score before and after psychological intervention}

Before and after intervention, the GAD-7 score and PHQ9 score were negatively correlated with the PC score but positively correlated with the NC score (Tables 4 and 5).

\section{Discussion}

Recently, the World Health Organization (WHO) listed COVID-19 as an international health emergency (7). According to China's valuable experience in dealing with COVID-19, in the absence of an effective vaccine for mass immunization, the most basic method to control infectious diseases (cutting off the route of transmission) is the most effective strategy to control the spread of the epidemic. For example, the Chinese government advocates that masks must be worn in public places, shelter hospitals in areas with more confirmed cases, and isolation points for isolation and treatment of suspected patients all over the country are effective guarantees for the rapid control of the epidemic in China.

During the isolation period, patients with suspected COVID-19 are separated from their families, lose communication with the outside world, and have insufficient social support. These factors, together with a lack of awareness of the disease, as well as the atmosphere of the isolation ward and the effects of medical staff wearing strict protective clothing, can provoke negative psychological reactions in patients $(8,9)$. At the same time, patients worry about themselves, their family members, and friends being infected, as well as the development and prognosis of the disease and the impact on their work; thus, they are prone to experiencing psychological issues such as anxiety, depression, and fear (10-13). Anxiety can manifest in patients as restlessness, irritability, and in severe cases, a rapid pulse, heavy breathing, insomnia, headache, and irritable. Depression can manifest as loss of interest, a depressed mood, crying, a sense of despair, sleep disturbance, loss of appetite, and decreased concentration (14). Excessive anxiety and panic are extremely detrimental to 
Table 2 Comparison of GAD-7 score and PHQ-9 score before and after psychological nursing intervention

\begin{tabular}{|c|c|c|c|c|c|c|}
\hline Group & \multicolumn{3}{|c|}{ GAD-7 } & \multicolumn{3}{|c|}{ PHQ-9 } \\
\hline Before intervention & $1.75 \pm 1.48$ & $7.26 \pm 1.18$ & $13.86 \pm 2.70$ & $2.38 \pm 1.31$ & $6.86 \pm 1.24$ & $13.82 \pm 3.52$ \\
\hline After intervention & $1.45 \pm 0.85$ & $4.31 \pm 1.15$ & $8.36 \pm 3.26$ & $1.38 \pm 0.82$ & $3.33 \pm 1.71$ & $7.76 \pm 3.42$ \\
\hline
\end{tabular}

GAD-7, Generalized Anxiety Disorder-7; PHQ-9, Patient Health Questionnaire-9.

Table 3 Comparison of TCSQ score before and after psychological nursing intervention

\begin{tabular}{lcc}
\hline Group & PC score & NC score \\
\hline Before intervention & $31.07 \pm 5.25$ & $28.78 \pm 6.72$ \\
After intervention & $36.40 \pm 5.93$ & $24.60 \pm 5.99$ \\
$t$ & 7.85 & 5.65 \\
$P$ & $<0.01$ & $<0.01$ \\
\hline
\end{tabular}

TCSQ, Trait Coping Style Questionnaire; PC, positive coping; NC, negative coping.

Table 4 Correlation analysis of GAD-7 total score, PHQ-9 total score and TCSQ score before psychological intervention

\begin{tabular}{lcc}
\hline Variable & PC score & NC score \\
\hline GAD-7 total score & & \\
$r$ & -0.719 & 0.741 \\
P & $<0.01$ & $<0.01$ \\
PHQ-9 total score & & \\
$r$ & -0.713 & 0.751 \\
P & $<0.01$ & $<0.01$ \\
\hline
\end{tabular}

GAD-7, Generalized Anxiety Disorder-7; PHQ-9, Patient Health Questionnaire-9; TCSQ, Trait Coping Style Questionnaire; PC, positive coping; NC, negative coping.negative coping.

patients' treatment and rehabilitation, and reduce the body's immunity (15), which is not conducive to recovery from the disease. At the same time, the above negative emotions also affect the treatment effect, and patients may even develop a distrust of medical staff, increasing the risk of medical disputes.

The results of this study showed that during isolation, the rates of anxiety and depression among patients with suspected COVID-19 before intervention were $46.72 \%$ and $48.20 \%$,
Table 5 Correlation analysis of GAD-7 total score, PHQ-9 total score and TCSQ score after psychological intervention

\begin{tabular}{lcc}
\hline Variable & PC score & NC score \\
\hline GAD-7 total score & & \\
$r$ & -0.733 & 0.687 \\
P & $<0.01$ & $<0.01$ \\
PHQ-9 total score & & \\
$r$ & -0.781 & 0.711 \\
$P$ & $<0.01$ & $<0.01$ \\
\hline
\end{tabular}

GAD-7, Generalized Anxiety Disorder-7; PHQ-9, Patient Health Questionnaire-9; TCSQ, Trait Coping Style Questionnaire; PC, positive coping; NC, negative coping.

respectively. However, after active intervention, the GAD7 and PHQ-9 scores were significantly lower than before intervention (all $\mathrm{P}<0.05$ ). Coping styles reflect individuals' stable coping behaviors and cognitive responses to various stress events. Positive coping styles can act as a buffer for the pressure caused by stress events. On the contrary, a negative coping style can aggravate the stress caused by a stress event. The results of this study showed that the positive coping score after intervention was significantly higher than that before intervention, while the negative coping score after intervention was significantly lower than that before intervention (all $\mathrm{P}<0.01$ ). Before and after intervention, GAD-7 score and PHQ-9 score were negatively correlated with $\mathrm{PC}$ score, but were positively correlated with $\mathrm{NC}$ score. The GAD-7 score and PHQ-9 score reflect the degree of anxiety and depression of isolated patients: the higher the GAD-7 and PHQ-9 scores, the higher the patient's NC score is likely to be, patients are more likely to respond negatively. Our observations show that the timely and effective implementation of psychological intervention methods can 
effectively relieve negative emotions in patients, change their negative emotions into positive ones, and relieve the pressure they feel, thus helping them to cope with the isolation period. In our study, a patient with normal GAD-7 score and PHQ9 score increased both scores (rated as mild anxiety and depression) after psychological nursing intervention, which was considered to be related to longer isolation time (6 days), recurrent fever and repeated pharynx swabs, suggesting that even patients with normal scores before intervention may have large fluctuations in their psychological state during the isolation period. This further shows the necessity and importance of psychological nursing interventions.

A new systematic review of the effects of COVID-19 on the mental state of medical staff and the general population, including 62 studies and 162,639 participants from 17 countries, found that the combined prevalence rates of anxiety and depression were $33 \%$ and $28 \%$, respectively. The prevalence rates of anxiety and depression were the highest among patients with previous diseases and COVID-19 infection (56\% and 55\%), and the situation of health care workers was similar to that of the general public. Common risk factors include women, nurses, low socioeconomic status, high risk of infection with COVID-19 and social isolation (16). The detection rates of anxiety and depression in this study were $46.72 \%$ and $48.20 \%$ respectively, similar to the conclusions of the above study. This study focuses on the influence of isolation state on the psychological status of suspected cases, and the improvement of patients' psychological status after taking corresponding psychological nursing intervention, which provides a new theoretical basis for evaluating the psychological status of isolated patients.

Compared with conventional nursing interventions, psychological nursing can reduce stress response and promote patient recovery (17). Through clinical practice, it can be concluded that positive psychological nursing interventions has the following advantages: (I) nurses understand and care about patients, achieve empathy, and establish a good nurse-patient relationship, which can relieve patients' anxious or depressive feelings (18), and improve their cooperation and compliance with treatment; (II) through positive education, patients' understanding of the disease can be improved, and the confidence they feel in overcoming the disease can be increased; (III) the use of professional and active communication methods, mobile phones, WeChat, and social media sites, along with reasonable use of non-verbal communication skills, can have a positive psychological impact on patients and drive positive emotions (19). Especially among adolescents, whose psychological and physiological development is not mature and is sensitive, psychological problems are widespread (20), and nursing interventions can alleviate adolescents' restless emotions. (IV) Effective psychological nursing can improve the patients' appetite and improve their resistance to disease.

To sum up, COVID-19 inflicts strong psychological stress on patients who are diagnosed or suspected to have the disease, which can result in psychological issues of varying degrees during isolation. An accurate evaluation of the psychological problems faced by patients during isolation and the implementation of positive intervention measures can help patients to face their disease. Interventions may include helping patients to adapt to the isolated environment as soon as possible, communicating to give positive guidance, showing concern for patients' psychological changes, and actively helping patients with social and emotional support. Such interventions can aid in obtaining the patient's trust and cooperation, and alleviating their negative feelings, thus helping them through the isolation period.

This study also has limitations and deficiencies, such as its sample small size. The cases included in this study are all patients around our hospital, and the number of study samples is greatly affected by the spread rate of the epidemic and government policy-making and other factors. If there is an opportunity to conduct further research, we will certainly try our best to enlarge the sample size and conduct a multicenter study to ensure the reliability of the research conclusions.

\section{Acknowledgments}

Funding: None.

\section{Footnote}

Reporting Checklist: The authors have completed the STROBE reporting checklist. Available at http://dx.doi. org/10.21037/apm-21-516

Data Sharing Statement: Available at http://dx.doi. org/10.21037/apm-21-516

Conflicts of Interest: All authors have completed the ICMJE uniform disclosure form (available at http://dx.doi. org/10.21037/apm-21-516). The authors have no conflicts of interest to declare.

Ethical Statement: The authors are accountable for all aspects of 
the work in ensuring that questions related to the accuracy or integrity of any part of the work are appropriately investigated and resolved. All procedures performed in this study involving human participants were in accordance with the Declaration of Helsinki (as revised in 2013). The study was approved by the Ethics Committee of Tianjin Third Central Hospital (No. bd2020111). A signed written informed consent agreement was provided by all patients before participation in the study.

Open Access Statement: This is an Open Access article distributed in accordance with the Creative Commons Attribution-NonCommercial-NoDerivs 4.0 International License (CC BY-NC-ND 4.0), which permits the noncommercial replication and distribution of the article with the strict proviso that no changes or edits are made and the original work is properly cited (including links to both the formal publication through the relevant DOI and the license). See: https://creativecommons.org/licenses/by-nc-nd/4.0/.

\section{References}

1. Lu R, Zhao X, Li J, et al. Genomic characterization and epidemiology of 2019 novel coronavirus: implications for virus origins and receptor binding. Lancet 2020;395:565-74.

2. Gorbalenya AE. Severe acute respiratory syndrome-related coronavirus- The species and its viruses, a statement of the Coronavirus Study Group. BioRxiv 2020.

3. Jiang $\mathrm{S}$, Shi Z, Shu $\mathrm{Y}$, et al. A distinct name is needed for the new coronavirus. Lancet 2020;395:949.

4. Ahorsu DK, Lin CY, Imani V, et al. Fear of COVID-19 Scale: Development and initial validation. Int J Ment Health Addict 2020:1-9.

5. Xiang YT, Yang Y, Li W, et al. Timely mental health care for the 2019 novel coronavirus outbreak is urgently needed. Lancet Psychiatry 2020;7:228-9.

6. Zandifar A, Badrfam R. Iranian mental health during the COVID-19 epidemic. Asian J Psychiatr 2020;51:101990.

7. Munster VJ, Koopmans M, van Doremalen N, et al. A Novel Coronavirus Emerging in China - Key Questions for Impact Assessment. N Engl J Med 2020;382:692-4.

8. Brooks SK, Webster RK, Smith LE, et al. The psychological impact of quarantine and how to reduce it: rapid review of the evidence. Lancet 2020;395:912-20.

9. Liu X, Luo WT, Li Y, et al. Psychological status and behavior changes of the public during the COVID-19 epidemic in China. Infect Dis Poverty 2020;9:58.

10. Tandon R. The COVID-19 pandemic, personal reflections on editorial responsibility. Asian J Psychiatr 2020;50:102100.

11. Geldsetzer P. Use of Rapid Online Surveys to Assess People's Perceptions During Infectious Disease Outbreaks: A Cross-sectional Survey on COVID-19. J Med Internet Res 2020;22:e18790.

12. Roy D, Tripathy S, Kar SK, et al. Study of knowledge, attitude, anxiety \& perceived mental healthcare need in Indian population during COVID-19 pandemic. Asian J Psychiatr 2020;51:102083.

13. Di Corrado D, Magnano P, Muzii B, et al. Effects of social distancing on psychological state and physical activity routines during the COVID-19 pandemic. Sport Sci Health 2020:1-6.

14. Wang C, Pan R, Wan X, et al. Immediate Psychological Responses and Associated Factors during the Initial Stage of the 2019 Coronavirus Disease (COVID-19) Epidemic among the General Population in China. Int J Environ Res Public Health 2020;17:1729.

15. Mitman G. Ebola in a stew of fear. N Engl J Med 2014;371:1763-5.

16. Luo M, Guo L, Yu M, Jiang W, Wang H. The psychological and mental impact of coronavirus disease 2019 (COVID-19) on medical staff and general public - A systematic review and meta-analysis. Psychiatry Res 2020;291:113190.

17. Cui DC, Geng J. Psychological stress and nursing strategies in patients with elective surgery. Zhonghua $\mathrm{Hu}$ Li Za Zhi 1996;31:692-5.

18. Botha E, Gwin T, Purpora C. The effectiveness of mindfulness based programs in reducing stress experienced by nurses in adult hospital settings: a systematic review of quantitative evidence protocol. JBI Database System Rev Implement Rep 2015;13:21-9.

19. Liu S, Yang L, Zhang C, et al. Online mental health services in China during the COVID-19 outbreak. Lancet Psychiatry 2020;7:e17-8.

20. Gyllenberg D, Marttila M, Sund R, et al. Temporal changes in the incidence of treated psychiatric and neurodevelopmental disorders during adolescence: an analysis of two national Finnish birth cohorts. Lancet Psychiatry 2018;5:227-36.

(English Language Editor: J. Reynolds)

Cite this article as: Niu W, Ma X, Zhang Y, Sun Z. The psychological effects of nursing interventions on patients with suspected COVID-19 during isolation. Ann Palliat Med 2021;10(6):6344-6350. doi: 10.21037/apm-21-516 